\title{
The Interactive Model of Theory and Practice in the Promotion of Science and Technology Quality of Chinese Kindergarten Teachers
}

\author{
Haiou Zhang ${ }^{1}$, Shijun $\mathrm{Xu}^{2 *}$ \\ ${ }^{1}$ The First author: Haiou Zhang (School of Education Science ,Anhui Normal University: No. 1 Beijing East Road, Wuhu, Anhui, China ) \\ The Second author (corresponding author) : Shijun Xu Telephone: 86+13772117072 Email:2934130805@qq.com Affiliation: School of Science, \\ Xi'an Technological University \\ Address: No.2 Xuefu Middle Road, Weiyang District, Xi'an, Shaanxi, China
}

\begin{abstract}
Chinese kindergarten teachers' low-level scientific-technological literacy has weakened the education of children's science field and even their teaching contents that have violated the principles and practices of science and technology. Taking the combination of theory and practice, from the top design to the educational practice, this paper studies the science and technology literacy of the kindergarten teachers, proposing three unique manifestations in the early childhood science education activities to create a circular interaction concept analogy to Qian Xuesen's thoughts of technical science, which conforms to the constructivist education activities of kindergarten teachers. The model of "interaction between theory and practice" is constructed based on this concept with the implementation strategy of "reducing theory" in science and technology theory and "swelling practice" in scientific and technological experience though academic education and continuing education using resources of colleges, society and network. This model which has achieved good results in three years of practice provides a reference model for improving the sciencetechnology literacy for kindergarten teachers in developing countries whose national conditions is similar with China.
\end{abstract}

Keywords: preschool teachers; scientific-technological literacy; interaction; theory and practice

\section{Introduction}

Either for the whole world or China, the development of science and technology plays an important or even decisive role in the development of the country. However, the key to the development of science and technology, which requires all aspects of education to focus on the cultivation of scientific and technological literacy and innovation, lies in the cultivation of innovative talents rather than just simple policies (Jiang, 1997; Ni et al.,2009; Taylor et al.,2008). At the initial stage of school education, science education in early childhood is crucial to their growth of innovation. Because of the scientific thinking and scientific and technological interest promoted by science and technology education at this stage, it will play a sustained and original role in talent growth (Eshach et al.,2005; Trnova et al.2015). China has entered a new era of high-quality development. The preschool education in the new era requires preschool teachers not only have a high quality of education, but also have high scientific and technological qualities(Xi,2017).

Generally, due to the low level of science-technology related literacy of preschool teachers in China (Zhou, 2012; Li,2012), kindergarten teachers have been weakened and even have abandoned the teaching of science (Wang,2008), which makes the science education in early childhood ineffective, while the traditional academic education and training model is difficult to work in the past two decades(Wang,2007). The researches and practices on the improvement of science-technology related literacy of kindergarten teachers are insufficient. It was found that in an educational activity of a well-known kindergarten in China, there were many teaching contents and examples that violate scientific principles and do not conform to scientific and technological facts (Zhang, 2017). Even experts in the field of pre-school education

Copyright (C) 2020 Haiou Zhang et al.

doi: 10.18282/le.v9i5.1191

This is an open-access article distributed under the terms of the Creative Commons Attribution Non-Commercial License

(http://creativecommons.org/licenses/by-nc/4.0/), which permits unrestricted non-commercial use, distribution, and reproduction in any medium, provided the original work is properly cited. 
have no active awareness of this issue. While in many general early childhood education institutions, the science-technology related literacy of teachers is generally too low to carry out standardized early childhood science education activities. Qualitative method is used to study the types and characteristics of preschool teachers' scientific education knowledge in order to help teachers realize what they have, and point out that it is necessary to develop from the perspective of dynamic development (Yang et al., 2009). Subsequently, a high-performance training model was proposed for the professional literacy improvement of preschool teachers (Jin,2012). Educational goals, contents, methods, evaluations, and other "children" solutions have been proposed to solve the current problems of kindergarten science education, one-sided attention to children's intellectual development, and emphasis on teachers' leadership (Li,2010). Not only that, in the study of improving the science-technology related literacy of kindergarten teachers, it also proposes a teacher-based development, which aims to inspire teachers' intrinsic motivation, pay attention to the participation experience of the whole process of training for trainees, and establish a new model of long-term training system (Yu, 2012). However, the above limited researches and practices are more of a theoretical description, lacking model construction, targeted strategies and approaches. Regarding the existing training models for kindergarten teachers, the science-technology related literacy training strategies have not been highlighted. Even if there are some new models, there is no effective implementation strategy.

\section{Research Background}

Chinese economy and population determine that teachers' education resources are far from sufficient. In general, the science-technology related literacy of Chinese kindergarten teachers are as follows:

\subsection{The overall science and technology foundation of Chinese kindergarten teachers is poor}

At present, most of these college students who choose pre-school majors are relatively poor-performed in junior high and senior high schools. They do not have the ability to high schools or colleges and thus choose this professional specialty. Most of the pre-school education students' learning of literature, history and the liberal arts received from the high school stage causes the asymmetry in the students' knowledge and knowledge structure. In addition, because of the drive-up goals, students majoring in liberal arts have no regard for the basics of natural science beyond mathematics. Although they have learned natural science courses, such as physics and chemistry in the junior high school, concepts of these courses are mostly specific and separate and laws are within their respective scopes. They do not pay enough attention to the concept of nature and unity of nature, let alone mastering scientific and technological methods and having a better understanding of scientific and technological thinking. In short, the scientific and technological knowledge is weak and the scientific and technological literacy is not good.

In the 21st century, with the rapid development of China's higher education, the kindergarten teacher education entered after graduating from junior high school gradually transitioned to the professional model school and higher teacher education after graduating from high school. Even so, the bachelor's degree and postgraduate education in 2008 accounted for only $9.28 \%$, and the vocational non-higher education accounted for $47.12 \%$ ( Pang et al,2012). Although the scale of schooling has been expanding in recent years, the quality of kindergarten students is worrying. Most of the students who choose pre-school teachers are students who are not performing well in the middle and high school entrance examinations. These students think that the knowledge of early childhood education is low-level and simple, and their own learning. The ability and knowledge base are not enough. Their own learning quality and knowledge foundation are doubtful.

\subsection{Pre-service academic education and post-employment retraining of kindergarten teachers have limited effects on scientific and technological literacy}

First, the low level of science-technology related literacy of preschool teachers is significantly related to their lower academic qualifications (Liu,2013). Preschool teachers are basically trained by preschool teachers' schools (secondary normal schools). They pay more attention to the training of skills for young teachers, such as music, dance, painting, etc. Science education, especially science and technology education, is not valued, until graduation. The kindergarten teachers lack basic technical literacy, which seriously affects their scientific quality. 
Secondly, post-employment retraining activities have failed to improve the ability of preschool teachers achieving science education activities. There is no difference in performance in early childhood science education between participating in current after-service training and no participating in post-employment training (Su, 2014). Obtain higher diplomas rather than improving the ability of children's science education by various types of academic education that kindergarten teachers participate in. At present, most types of teachers' continuing education courses are still centered on disciplines and teacher education, with emphasis on theory and neglect of practice while the methods of learning are mostly passive accepted learning, ignoring discovery and construction in practical situations, which purpose is more the acquisition of academic qualifications. Therefore, how to base the characteristics of the kindergarten teachers to design suitable curriculum content and learning methods is the key point for improving the science-technology related literacy of kindergarten teachers.

\subsection{In view of the importance of science-technology related literacy and the uniqueness of preschool children, children's science and technology education has the following unique characteristics.}

Early childhood science education is the initial and basic stage for a person's future scientific and technological literacy. In the growth of young children, teachers need to build a bridge between children and technology based on their own daily experience. Due to the cognitive characteristics of young children, children's science education should not only conform to the rigor of science of science itself, but also need to be empirically and life-oriented (in accordance with the cognitive characteristics of young children), both of which are in the process of education. Compatibility and balance is one of the unique manifestations of early childhood science education. The material of science curriculum comes from the thousands of phenomena in life. New ideas, new technologies and new industries happen all the time, while how to effectively use these dynamic curriculum resources, adapting to various new appearances and generating courses according to children's interests in a timely manner, this requires teachers to have pedagogical professional ability, scientific literacy and ability for continuing learning. This interdisciplinary comprehensive knowledge is the second manifestation of the uniqueness of early childhood science education. Because of the differences in the individual and growing environment, it is necessary to take into account individuality and universalization in the implementation of the concept of teaching in accordance with their ability. This is the third manifestation of the uniqueness of early childhood science education.

Then as a preschool teacher, the science-technology related literacy is directly related to the quality of the science curriculum for preschool children. Constructivism regard teachers in the classroom as the role of presenters, observers, questioners and designers, environmental organizers, public relations managers, children's learning archivists, classroom culture contributors, theoretical constructors, etc. which all preschool teachers are responsible for ( Chaille, et al.,2006). Therefore, the effect of the course is not only influenced by the course itself, but also by the self-study of the preschool teachers.

Based on the unique characteristics mentioned above, the idea about scientific nature of teachers will significantly affect their teaching performance. Science teachers are representatives of the scientific community in the classroom while teachers' words and deeds have a subtle influence (Palmer,2016; Gomes et al.,2017), not to mention children's strong teacher-oriented nature. Therefore, teachers' scientific literacy has a crucial influence on the effect of early childhood science education(National Research Council,1999) ( the scientific nature of teachers' knowledge and the logic of thinking all affect the formation of young children's scientific thinking and the generation of scientific interest(Zhang,2012; Slaÿana,2016; Berit,2016; Forawi,2016). In short, what kind of unique characteristics will decides what kind of scientific education effect.

\section{Theoretical Basis}

\subsection{Top design: Improving the science-technology related literacy of preschool teachers is an independent project rather than a dependency}

Considering the three unique manifestations of early childhood science education and the key role of preschool 
teachers, it is necessary to study top-level design in the whole process, model construction and educational practice for the problems of poor science-technology related literacy of Chinese kindergarten teachers. Because the science-technology related literacy of kindergarten teachers will eventually fall into the activities of early childhood education, it is manifested in: guiding young children to discover the mysteries of nature, exploring nature, paying attention to technology rather than presenting, encouraging children to explore in life scenes, letting young children to science and technology workers. The interest in exploration, research and development has always been a curiosity.

Qian Xuesen's scientific and technological thought

Qian Xuesen (Ma,2014) pointed out that natural science activities are the theoretical basis of engineering and technical activities. Engineering and technical activities are the application of natural science activities, but the application of natural science to engineering technology combination of scientific theory and engineering technology is a highly difficult and creative work, more appropriately, instead of a simply applied and deductive work. This scientifically based engineering theory is not the natural science itself, nor the engineering technology itself, but an independent discipline between natural science and engineering technology, namely technical science, as shown in Figure 1. This idea can be understood as a media-based technical science activity.

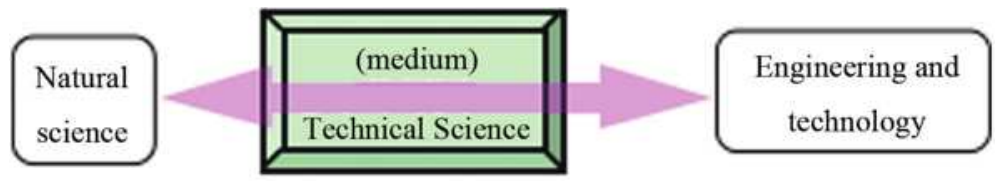

Fig.1. Qian Xuesen's thoughts on technical

\subsection{The concept of circular interaction in early childhood science education activities - in line with constructivism}

Early childhood science education activities are not simply to transfer scientific and technological knowledge to early childhood classrooms, nor to add scientific common sense to the implementation of early childhood curriculum. Technical knowledge, logical thinking and scientific methods are infiltrated into all aspects of early childhood education based on the law of children's psychological development which is done creatively by preschool teachers.

Analogy Qian Xuesen used the idea of technical science to link natural science activities and engineering technology activities, the concept of circular interaction between science and technology is proposed associated with early childhood science education activities, as shown in Figure 2. Early childhood science education is an independent medium, which combines scientific and technological activities with early childhood education activities. Science and technology activities "go" to early childhood education practice to enhance the scientific nature of early childhood science education activities while early childhood education activities "go" to early childhood science education activities to make children's science education activities more childification. The bilateral interactive cycle of early childhood education activities and scientific and technological activities is achieved by the science and technology education activities of kindergarten teachers.

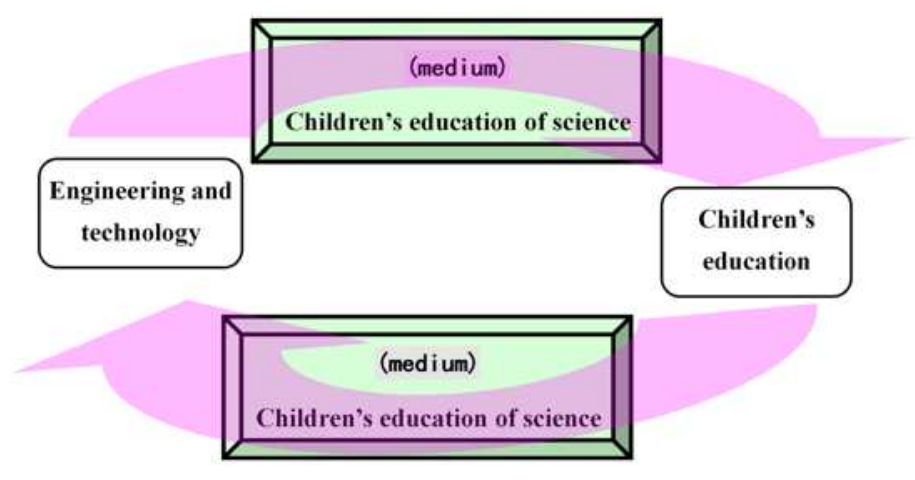

Fig.2. The concept of interaction

This concept has the following requirements for the creative work of kindergarten teachers in early childhood science education activities: according with the existing experience of young children, the phenomenon full of abstract and 
logical knowledge of adult world is designed to suitable curriculum based on the cognitive characteristics of young children which need implementation skills and good technical literacy.

\subsection{Interaction between Theory and Practice}

Guided by the circular interaction concept of preschool teachers' scientific and technological literacy improvement, aiming at the development of preschool teachers, from the interaction of theoretical cognition and practical experience, guarantying of various educational resources, the theory and practice of building lifting mode: bilateral interaction between theory and practice, and its implementation path and strategy which are shown in Figure.3

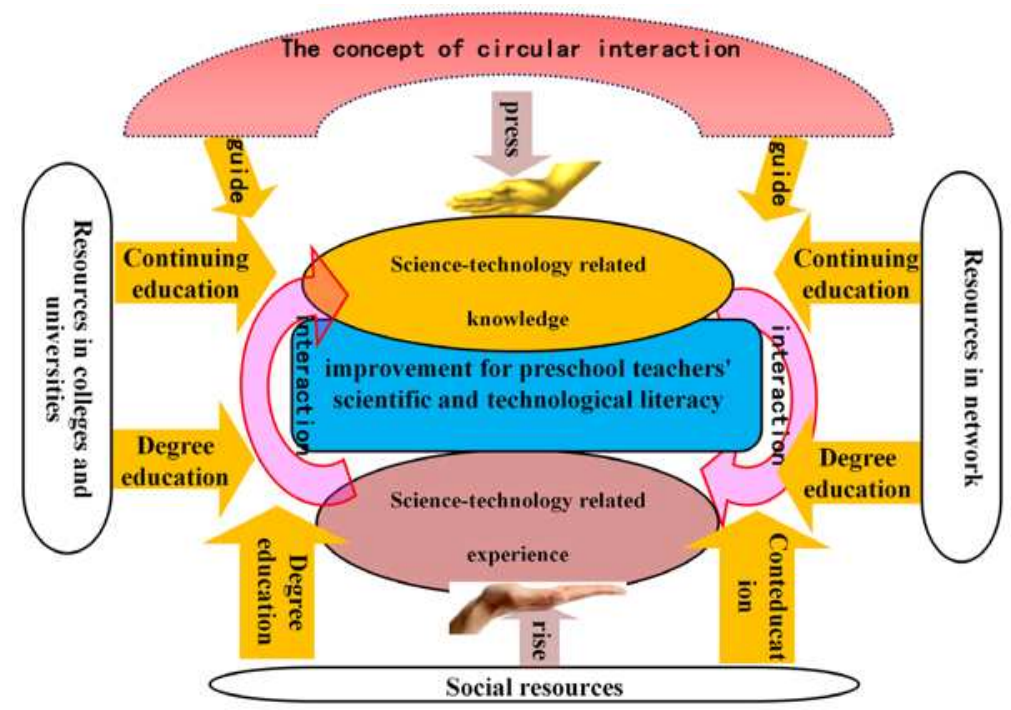

Fig.3. Enhancement mode: the interaction between theory and practice

Enhancement Mode: The Interaction between Theory and Practice

The concept of interaction is implemented in the science-technology related literacy promotion activities of kindergarten teachers. On the one hand, under the premise of ensuring the systematic and scientific nature of science and technology theory, reduce the difficulty and height of the existing scientific and technological theories which kindergarten teachers learn in order to improve the cognition of science and technology for kindergarten teachers. On the other hand, relying on social resources, university resources, network resources, etc., promote the cyclical interaction between the science and technology cognition of the kindergarten teachers and the technological experience through academic education, continuing education and other means for elevating the sublimation of experience and realizing the combination and interaction of its "technical theory cognition" and "scientific and technological practice experience". Through this model, we finally realize the improvement of science-technology related literacy of kindergarten teachers from the three dimensions of "science and technology theory knowledge", "scientific inquiry method and process" and "scientific interest and experience.

\section{Implementation strategy and approach}

\subsection{Implementation strategy}

Starting from the two sides of science and technology theory and practice experience of kindergarten teachers, on the one hand, reduce the difficulty of scientific and technological theory learning, connect with reality, increase application and interest, and promote the effective study of science and technology theory and knowledge by kindergarten teachers with liberal arts background, so as to better grasp children. Help kindergarten teachers dare to face the implementation of early childhood science education. On the other hand, increase the depth and breadth of the experience of science and technology, promoting the sublimation of empirical cognition from life, application and experience, and construct features transform knowledge, realizing the integration of practice and theory.

\subsection{Reducing Cognition of scientific and technological theory}

The possession of systematic scientific and theoretical knowledge is the starting point for the improvement of 
the science-technology related literacy of kindergarten teachers. Einstein believed that "whether you can observe the phenomenon at hand depends on what theory you use, and the theory determines what you can observe". In reality, kindergarten teachers often neglect the phenomenon of science and technology in life because the depth and breadth of science field is too difficult to understand. The reason why the kindergarten teachers reduce or even abandon the science and technology education activities, and narrowly understand science education as mathematics education is that the preschool teachers lack scientific and technological theories .

In the past, talent training for a long time, there is no difference between the kindergarten teachers and other personnel in learning the content, mode, system and requirements of scientific and technological theoretical knowledge, which leads to the low level of science and technology in kindergarten teachers. The scientific requirements, the technical principles and other scientific and technological theories, which will need to be reduced, so that the science and technology theory from the higher education institutions, textbooks, expert laboratories to life and become easier for the kindergarten teacher to understand scientific facts, phenomena, and laws. It is more willing to comprehend the scientific spirit and more fully grasp the scientific methods.

The scientific and technological theory recognizes the operational recommendations of "falling down". How to transfer complex scientific and technological theories to the kindergarten teachers clearly and concisely, and to make the theory go to life? According to Vygotsky's theory of recent development zones on individual development (real development level and potential development level), we choose to reduce the complexity of the complex scientific knowledge. In the relevant courses and trainings for improving the science-technology related literacy of kindergarten teachers, make the potential level of teachers turn into a realistic level of development, and the theory fall to the height that the kindergarten teachers can get. The "fall" is not fragmenting and simplifying the theory of science and technology roughly but keep the systematic study of scientific and technological theories. The purpose of examination and mathematical logic are diminished while the framework of the theoretical system of science and technology is generally grasped. When the logic operation is diluted, not all of accuracy is abandoned, but the key that description of the amount of nodes is retained.

For example, in learning optical knowledge, kindergarten teachers may not learn complex content such as Fourier transform and Laplace transform (this quantitative analysis is given to professional learners), but kindergarten teachers should understand the basic characteristics of light, such as sunlight is a kind of the complex light which is composed of several colors of light, and the colors are different because of the different wavelengths of light. The research of this subject found that in early childhood education, in the face of the rainbow theme the kindergarten teacher does not need to calculate the angle of the light of different color light during the formation of the rainbow shape, but it should be known that the formation of the rainbow shape is caused by the different wavelengths in the refraction and reflection. There are different paths that eventually form different circles or arcs. Understand the basic characteristics of light, in the existing shadow-based scientific activities, the kindergarten teacher can also guide children to discover the reflection and refraction of light. In short, kindergarten teachers can learn science and technology theory from the perspective of humanistic spirit, qualitatively learning scientific and technological knowledge, and improve their own scientific and technological literacy.

\subsection{Raising the experience of science and technology practice}

At present, the development of science and technology theory focuses on the exploration and application of universal and abstract scientific laws, is parallel with the development with the science and technology practice experience activities of kindergarten teachers, lack of communication and gaps, which makes the scientific and technological theoretical research far away from the kindergarten teachers' experience of science and technology practice. Therefore, it is necessary to increase the depth and breadth of the science and technology experience from kindergarten teachers, and to promote the sublimation of empirical cognition from life, application and experience which will help the improvement of science-technology related literacy of kindergarten teachers and the integration of practice and theory.

The improvement of the science-technology related literacy of kindergarten teachers cannot stay in the acquisition and understanding of scientific and technological theories but further self-digestion is needed. Only by effectively incorporating the scientific and technological theories and the practical experience of science and technology into the 
thinking, the preschool teachers can construct their own logic and systems in order to apply the basic theories they have learned. The experience of scientific and technological practice must be related to the theory of science and technology. Otherwise, the experience of science and technology, which is complicated, redundant and system-free, will increase the learning burden of the kindergarten teachers and discourage their enthusiasm for learning. In the training, not only the kindergarten teachers should be presented with various scientific and interesting phenomena, but also the scientific knowledge and principles contained in various phenomena should be explained scientifically and concisely.

Raise the scientific and practical experience of the operation recommendations. Deepen the depth and breadth of various science and technology practice experiences of kindergarten teachers, and raise them into deep understanding to create localized and characteristic courses, which is also needing first-line kindergarten teachers sharply capture scientific phenomena in teaching practice, getting the essence through phenomena, and then effectively use scientific and technological theories to guide practice.

For example, in view of the phenomenon of children slipping, if the kindergarten teacher can accurately focus the slip phenomenon around key knowledge points such as friction, teachers help children explore the cause of the slip with the child (observing the sole pattern, touching door handle and sole, associating slip experience, design related experiments, and perceived motion relationship), and generate a series of friction courses, teaching cases and their extended applications (such as safety education), getting rid of the limitations of inherent science education activities.

\section{Resources and ways to improve the science-technology related literacy of preschool teachers}

\subsection{Utilization of three types of resources}

1.Among the various types of colleges and universities in China, there are abundant and diverse courses and other resources covering various disciplines of arts, culture, industry, and agriculture. Pre-school teachers can make full use of various resources of the university in their pre-service academic education to improve their basic science-technology related literacy. The in-service teachers can choose their own science-technology resources according to their needs, then go to the university campus to experience the fun laboratory, participate in science-technology activities and update knowledge and ideas.

2.There are abundant social resources such as science-technology museums, research institutes and experience museums with science-technology knowledge and scientific-technological atmosphere where is interesting and it will be loved by kindergarten teachers and children. When training, try to explain the nature of science-technology lying in the phenomenon and realize the combination of theory and sensibility rather than stay at the surface of the fun phenomenon.

3. In the era of "Internet +", resources such as online courses, distance education and new learning methods have broken the limitations of time and space, realizing free and independent learning which create the new engine for kindergarten teachers'learn no matter pre-service and post-employment. Since 2012, large-scale online open courses have become popular in colleges and universities around the world, which have had an important impact on global higher education. Nowadays, network resources such as MOOCs, Spoc and online courses are generally accepted for all types of learning. For example, American universities have launched the three major MOOC platforms, Coursera, EDX and Udicity, attracting many famous universities from all over the world to open high-quality online education resources and services to global learners. In 2013, 109 famous universities opened 679 courses on this platform, 7696 thousand students enrolled in the platform to study(Larry Johnson,et al. 2013). Many well-known universities in China have joined the MOOC platform to build a global online course network with world-class universities such as Harvard, Stanford, Yale, and MIT.

\subsection{After-service education and training channels under various resources}

In academic education, colleges and universities set general foundation course, university physics and other natural science compulsory subjects which can meet the needs of kindergarten teachers to learn scientific -theoretical knowledge), and also have a large number of science and technology lectures, reports, science-technology competitions, 
science-technology practice activities, fun elective courses (suitable for the development of preschool teachers). Accepting the theoretical curriculum education helps the kindergarten teachers to consolidate the theoretical basis of science-technology, and participate in various science-technology practice activities so that the kindergarten teachers can apply the theory to practice. Through this way, such circular interaction can continuously improve the sciencetechnology related of literacy of kindergarten teachers.

In the non-academic education and training approach, kindergarten teachers can use colleges, networks, and social resources. TED, Icourse and other online open courses online are rich in resources covering a wide range of disciplines and break the space and time constraints for early childhood teachers. Following the principles of the recent development zone, you can set up a curriculum suitable for kindergarten teachers. With this platform, kindergarten teachers can flexibly arrange according to their own time, interests and needs, learning basic scientific knowledge, and expand practical applications.

\section{Practice and effect of the new model}

In the face of the low status of the science and technology literacy of kindergarten teachers and their poor cultivation effects, the research team took action supported by the project in the Shaanxi Provincial Preschool Education Research Major Project and the Teaching Research Project of the University Physics Curriculum Teaching Steering Committee of the Ministry of Education. The concept of interaction has constructed a "two-way interaction between theory and practice" to improve the science and technology literacy of kindergarten teachers. From 2016 to 2019, relying on the two project cooperation kindergartens, it took more than one year of training for 20 kindergarten teachers an and strategies such as "down one drop" and "rise one liter" had been implemented. The new model is further affecting the ability of kindergarten teachers to implement in educational activities in the field of early childhood science. .

The implementation effect of this model has been affirmed by the project cooperation kindergarten, and the main comparative observation indicators have been significantly improved. The number of winners of the "Best Teacher", "Innovation Award" and "Most Contribution Award" among the kindergarten teachers reached fourteen compared with those who did not participate in the model increased by 50\%. Some teachers in the training commented that "the real science education is not that pick up the leaves from the natural phenomenon presenting to the children, but guide the children to discover the mysteries of nature, explore nature, pay attention to technology. Take the familiar scenes in life as a starting point and give the children an freed environment where young children become interested in the exploration, research and development of science-technology workers, and always keep curiosity."

\section{Conclusion}

Aiming at the general situation of early childhood education in China, this paper puts forward three unique manifestations of early childhood science education activities, analogy to the way of Qian Xuesen's technical scientific thoughts, and puts forward the concept of circular interaction of kindergarten teachers' educational activities, and under the guidance of this concept, design The model of improving the science-technology related literacy of preschool teachers the "two-way interactive cycle of theory and practice", suggesting the practical strategy of "reducing one drop" and the experience of science and technology practice "up and down", as well as universities, society, and networks. The specific implementation of the three major resource utilization, and achieved good results in the three years of practice, significantly improved the science-technology related literacy of kindergarten teachers.

In short, the new model pointed out that the education and training for the improvement of science-technology related literacy of kindergarten teachers should proceed from the two aspects of scientific and technological theory and scientific and practical experience, and carry out interaction and promotion. While learning the universal science and technology theory, strengthen application, practice, experience and generation. New cognition, re-experience, recurring and spiraling, and finally improve the science-technology related literacy of kindergarten teachers. At the same time, it also provides some experience for countries with similar national conditions as China.

Funding: We earnestly acknowledge the support of the key project of Xi' an social science planning fund (JY119) for the practical domain of this study; the assistance of data collection and statistical analyses from teaching research 
project of Xi' an Technological University (18JGZ02); as well as the funding support from the soft science and social development project of Weiyang District of Xi' an (201944).

\section{Reference}

1. Taylor, A.R., Jones, M.G., Broadwell, B., Oppewal, T.(2008).Creativity, inquiry, or accountability? Scientists' and teachers' perceptions of science education.ScienceEducation,92 (6):1058-1075.

2. Chaille,C. ,Britain,L. (2006).Children as scientists: a constructivist approach to children's science education Translated by Xiao Yi, Liang Yuhua, Sun Wei. Beijing: Beijing Normal University Press.

3. Chen X.M.(2000). Qualitative research methods and social science research. Beijing: Education Science Press.

4. Palmer, E.F.A. (2016). How scientific concepts come to matter in early childhood curriculum: rethinking the concept of force. Cult Stud of Sci Educ,11(4): 1201-1222.

5. Trnova, E., Trna, J.(2015) .Formation of science concepts in pre-school science education[J]. Procedia-social and Behavioral Sciences, 197: 2339-2346

6. Eshach, H., Fried, M. N. (2005). Should science be taught in early childhood[J]. Journal of Science Education and Technology, (3):315-336

7. Jiang ,Z.M.(1997). Report of the 15th National Congress of the Communist Party of China . Beijing: People's Publishing House.

8. Jin, Y..(2012). Research on the Theory and Countermeasure of Improving the Professional Quality of Preschool Teachers. Adult Education, (1):61-62.

9. Gomes, J., Fleer, M.(2017).The development of a scientific motive:How preschool science and home play reciprocally contribute to science learning. Res Sci Educ,,(1):1-22

10. Johnson, L.et al(2013). NMC Horizon Report(2013Higher Education Edition). Austin: The New Media Consortium, 10: 11-14.

11. Ma,W.X.(2014).Interpretation of Qian Xuesen's Innovative Education Thought[M].Beijing: Higher Education Press,.9

12. National Research Council.(1999). National Science Education Standards. Translated by Ji Shouzhi et al. Beijing: Science and Technology Literature Publishing House.

13. Ni, G.W., Wang, Y.S.(2009). Physics and Culture . Beijing: Higher Education Press.

14. Li,Y.P.(2010). Scientific Literacy of Preschool Education Students. Culture and History Vision,(3):82-83

15. Li,Y.P. (2010). The Problems and Solutions of Current Kindergarten Science Education. Studies in Preschool Education,(7):60-62.

16. Liu Z.L. (2013). China Preschool Education Development Report 2012. Beijing: Education Science Press.

17. Pang,L.J. ,Hong, X.M.(2012). China Preschool Education Development Report . Beijing: Beijing Normal University Press.

18. Forawi, S. A. (2016). Standard-based science education and critical thinking. Thinking Skills and Creativity ,20:5262

19. Su,G.M.(2014).Study on the Implementation of Curriculum in Kindergarten Science (pp.11) .Beijing: People's Publishing House.

20. Wang, S.J.(2007). Analysis of the scientific literacy of preschool teachers .Forum on Science and Technology in China,(3):140-144.

21. Wang,P.(2008). The Status Quo and Development Countermeasures of Scientific Literacy of Preschool Education Students in Normal Universities. Studies in Preschool Education, (3): 25-27

22. Xi,J.P.(2017). Decisive victory to build a well-off society in an all-round way to win a great victory in socialism with Chinese characteristics in the new era_-Report at the 19th National Congress of the Communist Party of China . Beijing: People's Publishing House.

23. Yang,C.X.,Pang Li,J.(2009).Types and Characteristics of Science Education Knowledge of Preschool Teachers. Studies in Preschool Education,(7):25-28. 
24. Yu,T.(2012).Exploration of Creating a New Model of Preschool Teacher Training.Adult Education,(8):84-85

25. Zhang, H.O.(2017). The formation mechanism of neon and rainbow and its application in the practice of early childhood education. Science \& Technology Vision,(11):114.

26. Zhou, J.F.(2012).Discussion on the training mode of different levels of early childhood science education teachers. China Adult Education,(9):91-93

27. Zhang ,X.B.(2012). Reflection and Construction of Kindergarten Science Course .Changchun: Northeast Normal University Press.

28. Živkoviü Slaÿana(2016). A model of critical thinking as an important attribute for success in the 21st century. Procedia-social and Behavioral Sciences ,232:102-108

29. Berit, Z. (2016). The contribution of different patterns of teachers' interactions to young children's experiences of democratic values during play. Investigative Journalism Education Consortium,,48:179-192 